\title{
Dorsal midline cutaneous stigmata associated with occult spinal dysraphism in pediatric pati- ents
}

Hyun Jung Sung, MD, Hyun-Seung Lee, MD, PhD

Department of Pediatrics, College of Medicine, The Catholic University of Korea, Seoul, Korea

Purpose: To investigate the prevalence of occult spinal dysraphism (OSD) and subsequent neurosurgery in pediatric patients with isolated or combined dorsal midline cutaneous stigmata with or without other congenital malformations.

Methods: We carried out a retrospective review of patients who underwent sonography or magnetic resonance imaging (MRI) for OSD because of suspicion of dorsal midline cutaneous stigmata (presumed to be a marker for OSD) between January 2012 and June 2017. Information about patient characteristics, physical examination findings, spinal ultrasound and MRI results, neurosurgical notes, and accompanying congenital anomalies was collected.

Results: Totally 250 patients (249 ultrasound and one MRI screening) were enrolled for analysis. Eleven patients underwent secondary MRI examinations. The prevalence of OSD confirmed by an MRI was $2.4 \%$ (6 patients including one MRI screening). Five patients (2\%) had tethered cord and underwent prophylactic neurosurgery, 3 of whom had a sacrococcygeal dimple and a fibrofatty mass. Prevalence of tethered cord increased as markers associated with a sacrococcygeal dimple increased ( $0.5 \%$ of the isolated marker group, $8.1 \%$ of the 2 -marker group, and $50 \%$ of the 3 -marker group). Incidence of OSD with surgical detethering in 17 other congenital anomaly patients was $11.8 \%$, which was higher than the $1.3 \%$ in 233 patients without other congenital anomalies.

Conclusion: Our results suggest that the presence of dorsal midline cutaneous stigmata, particularly fibrofatty masses, along with a sacrococcygeal dimple is associated with OSD or cord tethering requiring surgery. OSD should be suspected in patients with concurrent occurrence of other congenital anomalies.

Key words: Occult spinal dysraphism, Congenital Abnormality, Sacral dimple

\section{Introduction}

Spinal dysraphism is a continuum of various congenital anomalies resulting from incomplete neural-tube closure during the neurulation period when skin and central nervous system originate from the same ectoderm. ${ }^{1,2)} \mathrm{A}$ variety of dorsal midline cutaneous stigmata present visible markers of occult spinal dysraphism (OSD) ranging from limited to soft tissue or skeletal structures to spinal cord tethering. ${ }^{1-3)}$ The overlying skin stigmata can be shown in up to 86.3\% of OSD patients, ${ }^{4)}$ and 4.2\%-7.2\% of healthy children, in which simple sacral dimples account for $74 \% \%^{2,5-7)}$ Sacral dimples have an incidence of 1.8\%-4\% of all births. ${ }^{8,9)}$ According to the degree of association with OSD, skin stigmata can be classified into 3 categories: (1) low-risk markers of simple dimples and deviated gluteal folds; ${ }^{10)}(2)$ intermediate-risk markers of vascular discolorations; (3) high-risk markers of aplasia cutis/congenital scars, dermal sinus tract, nonsimple or more cephalic dimples, hypertrichosis, caudal appendages, and
Corresponding author: Hyun-Seung Lee, MD, PhD Department of Pediatrics, Uijeongbu St. Mary's Hospital, College of Medicine, The Catholic University of Korea, 271 Cheonbo-ro, Uijeongbu 11765 , Korea

Tel: +82-31-820-3590

Fax: +82-31-821-3108

E-mail: iamlidia@catholic.ac.kr https://orcid.org/0000-0002-5219-8384

Received: 28 June, 2018

Revised: 26 September, 2018

Accepted: 1 October, 2018
Copyright (C) 2019 by The Korean Pediatric Society

This is an open-access article distributed under the terms of the Creative Commons Attribution NonCommercial License (http://creativecommons.org/ licenses/by-nc/4.0/) which permits unrestricted noncommercial use, distribution, and reproduction in any medium, provided the original work is properly cited. 
fibrofatty masses. ${ }^{1,2)}$ For vascular discolorations, small anomalies are considered low-risk while large anomalies are deemed high-risk. ${ }^{11)}$ Despite not generally presenting overt neurological impairments particularly in early life, OSD can lead to neurologic deterioration due to cord tethering, infective or aseptic meningitis due to epithelial-lining sinuses or dermod/epidermoids, or neural compression effects due to growing mass lesions. ${ }^{1,12,13)}$ Guidances for early screening for OSD and timely managements are warranted to prevent these complications, although the natural course of OSD has not been fully understood yet. ${ }^{1,13,14)}$

Tethered spinal cord is the most common and significant condition requiring neurosurgery. It occurs largely in lipomyelomeningocele, filum terminale abnormalities, congenital dermal sinus, limited dorsal myeloschisis, and split-cord malformation. ${ }^{1,13,15)}$ This condition refers to physical stretching of the spinal cord by a stalk-like structure anchoring to the overlying adjacent or distant tissues or structures. ${ }^{13)}$ It can be described radiographically when the conus medullaris ends below the middle third of the L2, ${ }^{1)}$ L2-3 interspace, ${ }^{5,16)}$ or L3 body. ${ }^{10,17)}$

Ultrasonography has been used as a first-line imaging modality to screen for OSD up to approximately 3-4 months of age when a distinct acoustic window is provided by unossified, cartilaginous posterior elements of the spine. ${ }^{3,11}$ It is known as effective as magnetic resonance imaging (MRI), the investigation of choice for examining intraspinal pathology., ${ }^{3,11}$ OSD has been clinically more important during the last decade due to increased more subtle anomalies that are difficult to screen with steady decline of neuraltube defects due to improved maternal nutrition and progress in prenatal screening methods. ${ }^{13,18)}$

In Korea, 2 retrospective studies addressed more than 1 sacral skin stigmata linked to abnormal lumbar ultrasound results that indicated $\mathrm{OSD}^{19)}$ and sacral dimples with hair or deviated gluteal folds associated with increased urogenital abnormalities. ${ }^{20)}$ Another retrospective study of spinal ultrasonography for 230 infants with isolated sacral dimple identified 27 infants with low-lying spinal cord and one with a tethered cord. ${ }^{211}$

The objective of this study was to investigate the prevalence of OSD and subsequent neurosurgery in pediatric patients with isolated or combined dorsal midline cutaneous stigmata with or without other congenital malformations. We also discussed current opinions about dorsal midline skin dimples and tethered spinal cord in terms of pathological anatomy based on embryogenesis.

\section{Materials and methods}

This was a retrospective review of patients at the age of 6 years or less with suspected dorsal midline cutaneous stigmata who had been referred by primary care physicians or who were born in the hospital and underwent sonography or MRI evaluation for OSD at the pediatric department of Uijeongbu St. Mary's Hospital between January 1, 2012 and June 30, 2017. Dorsal midline cutaneous stigmata were defined as presumed markers for OSD. Study participants were identified based on a report generated by Uijeongbu St. Mary's Hospital medical record database. After excluding patients who had insufficient information for this study, the remaining subjects were assigned to the study population. Individual medical records were reviewed to collect the following data: patients' characteristics, physical examination findings of cutaneous markers of OSD, spinal ultrasound and MRI findings, neurosurgical referral and management notes, and other accompanying congenital anomalies. Dorsal midline skin lesions included the following: lumbar, sacral, or coccygeal dimples, deviated or duplicated gluteal furrows as abnormal folds split or with any other degree of deviation, hypertrichosis with long, silky hairs as opposed low-risk light ones, dermal or subcutaneous masses (acrochordon, fibroma, and lipoma), and vascular discolorations (infantile hemangioma, port-wine stains, and nevus flammeus). Pigmented lesions encompassing Mongolian spots were excluded. Spinal ultrasound findings were reviewed to determine skeletal structure, conus level, cauda equina symmetry, cord pulsatility and position, thickness of the filum terminale, and intrathecal masses. The following findings were considered to be highly indicative of tethered cord: conus medullaris terminating inferior than the L2-3 disc space with eccentric position of the cord, intrathecal mass or lipoma, dermal sinus tract across the soft and bony tissue continuous with intraspinal structures, and thickened or fatty filum terminale at more than $2 \mathrm{~mm}$ in width or with bulbous appearance. Findings, such as ventriculus terminalis and filar cyst were considered as normal variants. MRI findings were reviewed for the site of cutaneous dimples as well as for abnormal ultrasound findings. Ultrasonography was performed by radiologists using a Philips IU-22 equipment (Philips, Bothell, WA, USA) with a 12$\mathrm{MHz}$ linear transducer. MRI scan was conducted using 3.0 T MRI Magnetom (Verio, Siemens, Munich, Germany). T1 and T2 sagittal, axial, and coronal images at least up to the thoracic spine with image slice thickness ranging from $2.5 \mathrm{~mm}$ to $5 \mathrm{~mm}$, were obtained. This study was approved by the Institutional Review Board of Uijeongbu St. Mary's Hospital, The Catholic University of Korea (approval number: UC18RESI0050). Informed consent was waived by the board.

\section{Results}

Fig. 1 shows the flow of the study population. Table 1 lists characteristics of the study population. Medical records of 255 patients who were screened by sonography or MRI examinations for OSD because of suspicious dorsal midline cutaneous stigmata were reviewed. Five patients were excluded because of insufficient data recording of clinical information. The remaining 250 patients (249 ultrasound 


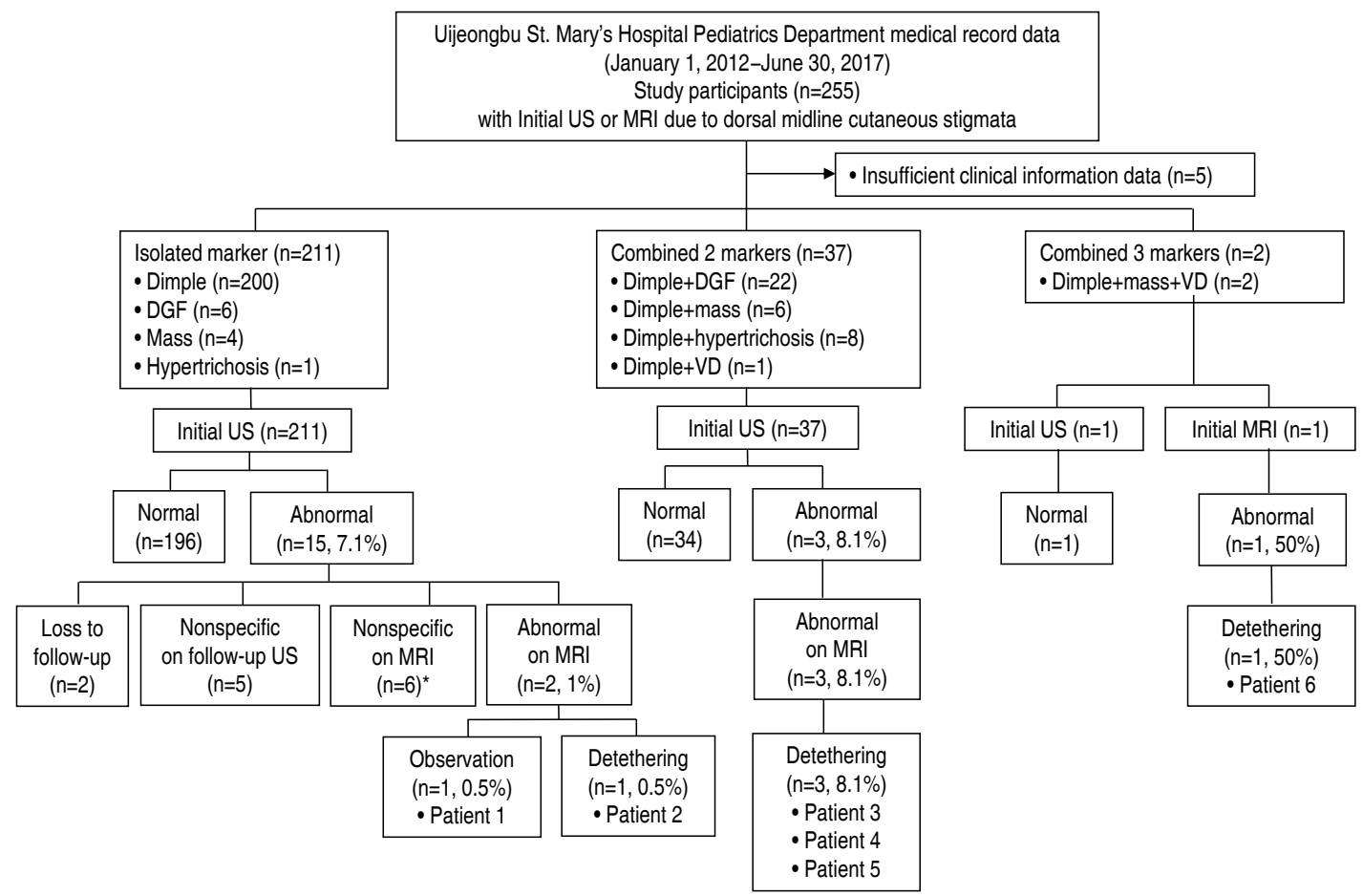

Fig. 1. Schematic depicting the study population. DGF, deviated or duplicated gluteal furrows; VD, vascular discoloration; US, ultrasonography; MRI, magnetic resonance imaging; OP, operation. *Note that these cases included one with a chromosomal anomaly and horseshoe kidney and one with a communicating hydrocele requiring surgery.

and one MRI screening) were enrolled for analysis of descriptive statistics. They were categorized into 3 groups according to the number of cutaneous markers: isolated marker group, combined 2-marker group, and combined 3-marker group.

Of 250 patients, 141 (56.4\%) were males. On physical examination for dorsal midline cutaneous markers, 239 (95.6\%) had single sacrococcygeal dimples. All cases were described as having single dimples located within sacral or coccygeal areas. However, not all records provided clear information of dimple size in width or depth, distance from the anal verge, or presence of visible drainage. Twenty -eight (11.2\%) had deviated or duplicated gluteal furrows, 12 (4.8\%) had cutaneous masses (seven with acrochordon and 5 with other dermal or subcutaneous masses), 9 (3.6\%) had hypertrichosis, and $3(1.2 \%)$ had vascular discolorations (2 with nevus flammeus and 1 with telangiectasia within the atrophic skin) (Table 1). A total of $211(84.4 \%)$ presented with isolated markers (200 with solitary dimples, 6 with deviated or duplicated gluteal furrows, 4 with cutaneous masses, and 1 with hypertrichosis). Thirty-seven (14.8\%) showed a combination of 2 markers (single dimples in common, deviated or duplicated gluteal furrows in 22, cutaneous masses in 6 , hypertrichosis in 8 , and a vascular discoloration in 1). Two had a combination of 3 markers (a dimple, cutaneous mass, and vascular discoloration) (Fig. 1). None had neurological manifestation. Seventeen (6.8\%) had other congenital malformations in addition to dorsal midline skin lesions (Table 1). Of these, 14 had isolated dimples.
One with communicating hydrocele that required surgery showed a dimple and hypertrichosis. One with craniosynostosis showed a dimple and deviated gluteal folds while one with atrial septal defect showed a dimple and subcutaneous lipoma. These 2 underwent neurosurgical intervention because of cord tethering (Fig. 1).

Initial screening imaging (249 subjects with sonography and 1 with MRI) was done at an average age of 30 days, ranging from 1 to 426 days. Four underwent sonography at more than 6 months of age (191, 191, 197, and 321 days of life, respectively) while 1 underwent initial MRI at 426 days of age (Table 1). Amongst these 249 subjects, 18 (7.2\%) showed abnormal spinal ultrasounds. They were referred to neurosurgical specialists. Of these 18 subjects, 5 (2\% of the 249) remained abnormal on subsequent MRI, 11 (4.4\%) turned out to be false-positive on follow-up sonography or MRI, and 2 ( 0.8 \%) were lost to follow-up (Fig. 1).

A total of 6 patients (2.4\%), including 1 with initial MRI, were confirmed to be abnormal on MRI, including 2 (1.0\%) of the isolated marker group, 3 (8.1\%) of the combined 2-marker group, and 1 (50\%) of the combined 3-marker group (Fig. 1). Of these, 5 patients underwent initial sonography, and the initial screening time was within 3 months, largely in one month.

Five subjects (2\%) underwent preventive tethered-cord release, including: (1) 1 (0.5\%) of the isolated marker group (a 19-day-old male with a sacral dimple who had a tethered cord and underwent detethering at 9 months of age); (2) 3 (8.1\%) of the combined two- 
marker group (a 3-month-old male with a coccygeal dimple and skin tag who had a tethered cord and lipomyelomeningocele and underwent detethering at 5 months of age; a 16-day-old male with a sacral dimple, deviated gluteal folds, and craniosynostosis who had a tethered cord and a dermal sinus tract with an intraspinal

\begin{tabular}{lc}
\multicolumn{2}{l}{ Table 1. Clinical characteristics of the study population $(\mathrm{n}=250)$} \\
\hline Characteristic & Value \\
\hline Age at initial examination (day) & $30(1-426)^{\star}$ \\
Gestational age (wk) & $38.7(29.9-41.3)$ \\
Birth weight (kg) & $3.03(1.25-4.33)$ \\
Male sex & $141(56.4)$ \\
Congenital malformations & $17(6.8)$ \\
Chromosomal anomaly & $5^{\dagger}(2.0)$ \\
Congenital heart disease & $4^{\ddagger}(1.6)$ \\
Cleft palate & $1(0.4)$ \\
Craniosynostosis & $1(0.4)$ \\
Renal agenesis, unilateral & $1^{\ddagger}(0.4)$ \\
Horseshoe kidney & $1^{\dagger}(0.4)$ \\
Urogenital anomaly requiring surgery & $6(2.4)$ \\
Dorsal midline cutaneous stigmata & \\
Sacrococcygeal dimple, single & $239(95.6)$ \\
Deviated or duplicated gluteal furrows & $28(11.2)$ \\
Cutaneous mass & $12(4.8)$ \\
Skin tag & $7(2.8)$ \\
Other dermal or subcutaneous mass & $5(2.0)$ \\
Hypertrichosis & $9(3.6)$ \\
Vascular discoloration & $3(1.2)$ \\
Nevus flammeus & $2(0.8)$ \\
Telangiectasia within the atrophic skin & $1(0.4)$ \\
\hline
\end{tabular}

Values are presented as mean (range) or number (\%).

*Four patients underwent ultrasonography screening at greater than 6 months of age $(191,191,197$, and 321 days of life, respectively) and one patient underwent initial magnetic resonance imaging at 426 days of age. Two cases had congenital anomalies concurrently: 'one with chromosomal anomaly and horseshoe kidney, and ${ }^{\ddagger}$ one with congenital heart disease and unilateral renal agenesis. communication and underwent detethering at 3 months of age; and a 2-day-old female with a coccygeal dimple, subcutaneous lipoma, and atrial septal defect who had a tethered cord and lipomyelomeningocele and underwent detethering at 3 months of age); and (3) 1 (50\%) of the combined 3-marker group (a 14-monthold female with a coccygeal dimple, subcutaneous lipoma, and nevus flammeus who had a tethered cord and fatty filum terminale and underwent detethering at 14 months of age) (Figs. 1, 2; Table 2). One patient with an isolated coccygeal dimple had a dermal sinus tract ending at the tip of the coccyx without intraspinal association (Fig. 1, Table 2). The prevalence of tethered cord in each dorsal midline stigma was as follows: 2.1\% (5 of 239 patients) with single

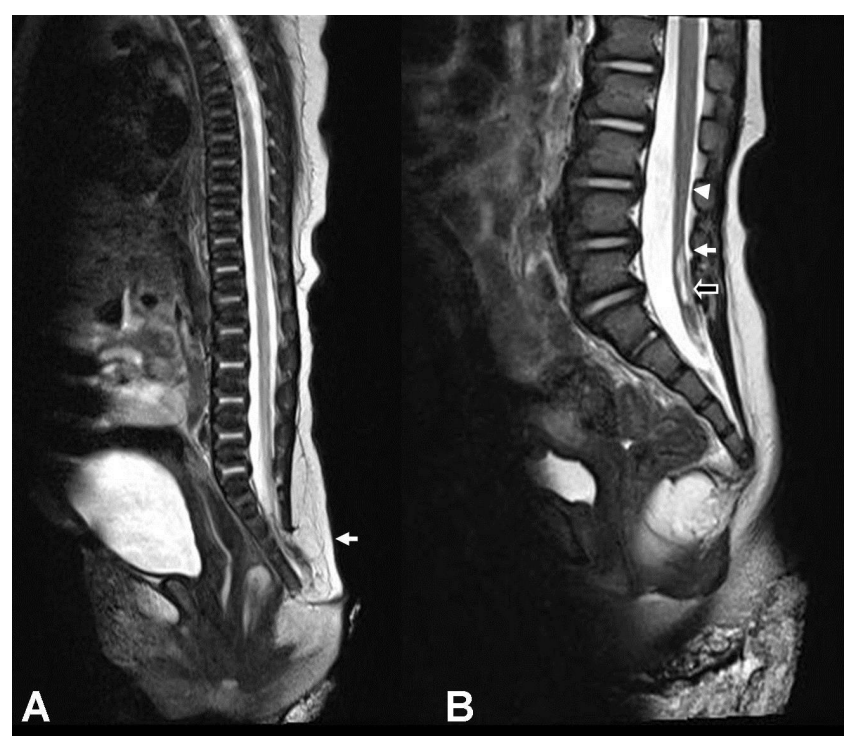

Fig. 2. Magnetic resonance (MR) imaging findings for patients 5 and 6 . (A) A T2-weighted MR image of patient 5 shows the conus medullaris at the sacral level and a subcutaneous fatty mass with lipomatous placode (arrow) connected to the cord through the spina bifida of the sacrum. (B) A T2-weighted MR image of patient 6 shows the conus medullaris at L3-4 (arrowhead) and fatty filum terminale (arrow) with an enhancing portion at S1-2 (open arrow).

Table 2. Patients with occult spinal dysraphism

\begin{tabular}{|c|c|c|c|c|c|}
\hline $\begin{array}{l}\text { Patients } \\
\text { No. }\end{array}$ & $\begin{array}{c}\text { Age at examination } \\
\text { and sex }\end{array}$ & $\begin{array}{c}\text { Dorsal midline } \\
\text { cutaneous stigmata }\end{array}$ & Congenital anomalies & Occult spinal dysraphism & Dethetering age \\
\hline 1 & 57-Day-old female & Coccygeal dimple & - & Dermal sinus tract ending at the tip of the coccyx & Not done \\
\hline 2 & 19-Day-old male & Sacral dimple & & Tethered cord & 9 Months \\
\hline \multirow[t]{2}{*}{3} & 3-Month-old male & Coccygeal dimple & - & Tethered cord & 5 Months \\
\hline & & Skin tag & - & Lipomyelomeningocele & \\
\hline \multirow[t]{2}{*}{4} & 16-Day-old male & Sacral dimple & Craniosynostosis & Tethered cord & 3 Months \\
\hline & & Deviated gluteal folds & & Dermal sinus tract & \\
\hline \multirow[t]{2}{*}{5} & 2-Day-old female & Coccygeal dimple & Atrial septal defect & Tethered cord & 3 Months \\
\hline & & Subcutaneous lipoma & & Lipomyelomeningocele & \\
\hline \multirow[t]{3}{*}{6} & 14-Month-old female & Coccygeal dimple & - & Tethered cord & 14 Months \\
\hline & & Subcutaneous lipoma & & Fatty filum terminale & \\
\hline & & Nevus flammeus & & & \\
\hline
\end{tabular}


sacrococcygeal dimples, 3.6\% (1 of 28) with deviated or duplicated gluteal furrows, 25\% (3 of 12) with masses, 0\% (0 of 9) with hypertrichosis, and 33.3\% (1 of 3) with vascular discolorations.

\section{Discussion}

The current study showed that the prevalence rate of OSD confirmed by MRI in pediatric patients who underwent initial spinal ultrasound and MRI because of suspected dorsal midline cutaneous stigmata was 2.4\% ( 6 of 250 patients). All had single sacrococcygeal dimples, isolated or combined with a fibrofatty mass, deviated gluteal folds, or a mass and a vascular lesion (Fig. 1, Table 2). Of these 6 patients, 5 (2\% of 250 patients) underwent prophylactic surgical untethering and 1 had a dermal sinus tract without any intraspinal connection (Fig. 1, Table 2). The prevalence of tethered cord elevated with increasing number of additional markers to a sacrococcygeal dimple: $0.5 \%$ in the isolated marker group almost consisting of isolated sacrococcygeal dimples, $8.1 \%$ in the combined group of 2 markers, and 50\% in the combined group of 3 markers (Fig. 1). Of the 5 patients who underwent surgery, 3 had a sacrococcygeal dimple and a fibrofatty mass: these patients account for $37.5 \%$ of the 8 patients with a dimple and a mass and $25.0 \%$ of the 12 patients with a mass (Fig. 1). These findings suggested that concurrent presence of more dorsal midline cutaneous markers, particularly a combination of a sacrococcygeal dimple and a dermal or subcutaneous fatty mass, was associated with cord tethering, in line with previous studies addressing multiple clinical indications for work-ups to detect OSD. ${ }^{14,22,23)}$

Our study showed rates of OSD and neurosurgery in relation to sacral dimples as follows: $2.4 \%$ and $2.1 \%$, respectively, of 239 patients with single sacrococcygeal dimples, and $1 \%$ and $0.5 \%$, respectively, in 200 isolated dimple patients (Fig. 1). A study of 3,884 healthy infants with a simple sacral dimple by Kucera et al. ${ }^{5)}$ reported a confirmed abnormal ultrasound rate of $2 \%$ and a neurosurgical intervention rate of $0.13 \%$. Other reports of ultrasound screening showed similar incidence of confirmed abnormal ultrasound and neurosurgery: 0 in 109 infants with simple dimples; ${ }^{10)} 0$ in 270 newborns with isolated simple dimples; $;^{22)} 6.0 \%$ and $0.8 \%$, respectively, in 638 infants with isolated sacral dimples; ${ }^{16)}$ and 5\% and 1\%, respectively, in 151 newborns with sacral dimples. ${ }^{12)}$

In light of pathological anatomy, 2 conditions posing surgical cord tethering with abnormal connections between the spinal cord and surrounding structures were found in our study: a dermal sinus tract associated with an overlying skin pit and fibrolipomatous stalks in lipomyelomeningocele and fatty film terminale associated with subcutaneous lipoma and acrochordon. Theoretically, OSD may present with embryologically derived cord-like structures associated with cord tethering, consisting of fibrous, epithelial, fatty, and neural elements. During primary neurulation period, incomplete dysjunction of cutaneous ectoderm and neuroectoderm may lead to epithelial-lined sinuses with overlying skin openings and fibroneural stalks in limited dorsal myeloschisis, which can pull down the overlying atretic skin pits. Premature dysjunction can result in mesoderm being trapped, which may lead to fibrofatty pedicles in lipomyelomeningocele with extending or associated fibrolipomatous masses in the skin and subcutaneous tissue. ${ }^{2,3,24)}$ In the secondary neurulation, incomplete involution of the distal spinal cord may lead to fatty or thickened filum terminale. ${ }^{3)}$ Hence, dorsal midline cutaneous stigmata including dimples and fatty masses have been regarded as significant markers for underlying OSD that can cause cord tethering or intraneural infective complication.

Dorsal midline skin dimples or pits may be lumbar, sacral, or coccygeal in location. ${ }^{1,9)}$ When located more caudally to the coccyx, dimples are considered to be innocent because the underlying fibrous tract traverses to the coccygeal tip without intraneural association, which may derive from caudal cell mass anomalies in the secondary neurulation. ${ }^{1,2,3,9)}$ However, the dimples more cranial than the middle sacrum are clinically important because they can possess 2 conditions and pose an abnormal intraneural connection that requires neurosurgical interventions: congenital dermal sinuses and limited dorsal myeloschisis. ${ }^{1,9)}$ The both lesions may result from the identical mechanism of embryonic pathogenesis and can appear as mixed in one lesion. ${ }^{15,25)}$

There have been controversies over dorsal midline cutaneous dimples because of their low value of predicting OSD. Prior studies termed benign, innocuous dimples "simple sacral dimples," which require no further imaging study, to distinguish from the more highly predictable dimples. ${ }^{9,26,27)}$ Simple dimple has been defined as a midline pit within the intergluteal crease, no greater than 2.5 $\mathrm{cm}$ above the anus, less than $5 \mathrm{~mm}$ in diameter, and as an solitary lesion without other associated cutaneous stigmata. ${ }^{6,10,26)}$ Current guidelines propose that neurosurgical referral and/or spinal MRI should be prompted when atypical or multiple dimples exist or when a sacral dimple is in conjunction with either additional cutaneous stigmata (hair tufts or hemangioma) or abnormal neurological manifestations. ${ }^{22,27)}$ However, the criteria for defining simple dimples developed a priori were based largely on retrospective studies without normal controls. This may give rise to ambiguity when applied in clinical practice that requires reassurance. For limited dorsal myeloschisis, a recently defined distinct form of OSD, the associated dorsal midline dimple may be small in diameter and not deep without an opening and may occur as an isolated lesion that simulates a simple dimple. ${ }^{15)}$ The site criterion distinguishing between simple and clinically significant atypical dimples is not clear for the upper border with the variability with age in definition. Furthermore, evidence of the presence of associated neural anomalies with isolated simple dimples has been found in the literature with very low ${ }^{5,10,12)}$ to relatively high occurrence rates. ${ }^{14,17)}$ Two studies of infants with MRI screening because of skin dimples 
revealed the occurrence of OSD at 22.8\% (33 of 145) in the group with simple dimples within $11 \mathrm{~mm}$ of the coccyx ${ }^{14)}$ and at 16.7\% (14 of 84) in intragluteal dimples. ${ }^{17)}$ Accordingly, a prospective study of dorsal midline skin dimples is warranted to have clear criteria to determine whether dimples are innocent or significant through pathological anatomy approaches. In our study, all coccygeal dimples were innocuous without intraspinal communication. In 200 cases of isolated sacrococcygeal dimples, only one cord tethering was found with a sacral dimple.

The association between congenital malformations other than dorsal midline cutaneous stigmata and OSD has been reported. ${ }^{3,12,16,17)}$ On the basis of these associations, Wilson et al. ${ }^{12)}$ have suggested that congenital anomalies in addition to cutaneous stigmata should be included as high-risk signatures in a screening guideline. Our study revealed that the incidence of OSD with detethering in 17 other congenital anomaly patients was $11.8 \%$, which was higher than the 1.3\% in 233 patients without other congenital anomalies.

Our diagnostic yield of 5 cases of OSD with initial sonography within 3 months correlated well with the clinical availability of ultrasound screening for OSD in early infancy. MRI can be recommended primarily when there are neurological manifestations, when contexts are unavailable for spinal ultrasound including older ages and anorectal malformations, ${ }^{28)}$ and when they are highly predictable constellations of clinical features including simultaneous presence of more cutaneous stigmata and/or other concurrent malformations. ${ }^{22)}$

Our study has limitations. A retrospective study is always prone to have biases, which warrant a prospective controlled study. Our investigators had to depend on several clinicians' documentations. Thus, sometimes we had insufficient or relatively unclear information. There might be potential risk for incorrect diagnosis on ultrasonography in four older subjects who had vertebral ossification (Table 1). The small size of the sample collected in one setting limits generalization of our results.

In conclusion, our results suggest that addition of other dorsal midline cutaneous stigmata, particularly fibrofatty masses, to a sacrococcygeal dimple is associated with OSD or cord tethering. Concurrent presence of other congenital anomalies should be considered a predictable condition for OSD.

\section{Conflicts of interest}

No potential conflict of interest relevant to this article was reported.

\section{References}

1. Dias M, Partington M; Section on Neurologic Surgery. Congenital brain and spinal cord malformations and their associated cutaneous markers. Pediatrics 2015;136:e1105-19.

2. Sewell MJ, Chiu YE, Drolet BA. Neural tube dysraphism: review of cutaneous markers and imaging. Pediatr Dermatol 2015;32:161-70.

3. Meyers AB, Chandra T, Epelman M. Sonographic spinal imaging of normal anatomy, pathology and magnetic growing rods in children. Pediatr Radiol 2017;47:1046-57.

4. Schropp C, Sörensen N, Collmann H, Krauss J. Cutaneous lesions in occult spinal dysraphism--correlation with intraspinal findings. Childs Nerv Syst 2006;22:125-31.

5. Kucera JN, Coley I, O'Hara S, Kosnik EJ, Coley BD. The simple sacral dimple: diagnostic yield of ultrasound in neonates. Pediatr Radiol 2015;45:211-6.

6. Kriss VM, Desai NS. Occult spinal dysraphism in neonates: assessment of high-risk cutaneous stigmata on sonography. AJR Am J Roentgenol 1998;171:1687-92.

7. Henriques JG, Pianetti G, Henriques KS, Costa P, Gusmão S. Minor skin lesions as markers of occult spinal dysraphisms--prospective study. Surg Neurol 2005;63 Suppl 1:S8-12.

8. Sarikaya Solak S, Kivanc Altunay I, Tukenmez Demirci G, Can B. Prevalence of congenital cutaneous anomalies in 1000 newborns and a review of the literature. Am J Perinatol 2016;33:79-83.

9. Weprin BE, Oakes WJ. Coccygeal pits. Pediatrics 2000;105:E69.

10. Ben-Sira L, Ponger P, Miller E, Beni-Adani L, Constantini S. Low-risk lumbar skin stigmata in infants: the role of ultrasound screening. J Pediatr 2009;155:864-9.

11. Ben-Sira L, Ponger P, Constantini S. Evaluation of dorsal midline discolorations with physical examination and ultrasound. J Pediatr 2017;190:246-50.

12. Wilson P, Hayes E, Barber A, Lohr J. Screening for spinal dysraphisms in newborns with sacral dimples. Clin Pediatr (Phila) 2016;55:106470.

13. Venkataramana NK. Spinal dysraphism. J Pediatr Neurosci 2011;6 (Suppl 1):S31-40.

14. O'Neill BR, Gallegos D, Herron A, Palmer C, Stence NV, Hankinson TC, et al. Use of magnetic resonance imaging to detect occult spinal dysraphism in infants. J Neurosurg Pediatr 2017;19:217-26.

15. Lee SM, Cheon JE, Choi YH, Kim IO, Kim WS, Cho HH, et al. Limited dorsal myeloschisis and congenital dermal sinus: comparison of clinical and MR imaging features. AJNR Am J Neuroradiol 2017;38:17682.

16. Chern JJ, Kirkman JL, Shannon CN, Tubbs RS, Stone JD, Royal SA, et al. Use of lumbar ultrasonography to detect occult spinal dysraphism. J Neurosurg Pediatr 2012;9:274-9.

17. Harada A, Nishiyama K, Yoshimura J, Sano M, Fujii Y. Intraspinal lesions associated with sacrococcygeal dimples. J Neurosurg Pediatr 2014;14:81-6.

18. Committee on Practice Bulletins-Obstetrics. Practice Bulletin No. 187: Neural Tube Defects. Obstet Gynecol 2017;130:e279-90.

19. Hong M, Lee YK, Ko SY, Shin SM, Han BH, Kim KA. Sacral cutaneous clues to underlying spinal abnormalities. Neonatal Med 2014;21:4651.

20. Kim YS, Jin DH, Shin JH, Lee EH, Choi BM, Hong YS, et al. Intraspinal and urogenital abnormalities in infants with sacral cutaneous lesions. Neonatal Med 2015;22:40-7.

21. Choi JH, Lee T, Kwon HH, You SK, Kang JW. Outcome of ultrasonographic imaging in infants with sacral dimple. Korean J Pediatr 2018; 61:194-9.

22. Ausili E, Maresca G, Massimi L, Morgante L, Romagnoli C, Rendeli C. Occult spinal dysraphisms in newborns with skin markers: role of ultrasonography and magnetic resonance imaging. Childs Nerv Syst 2018;34:285-91.

23. McGovern M, Mulligan S, Carney O, Wall D, Moylett E. Ultrasound 
investigation of sacral dimples and other stigmata of spinal dysraphism. Arch Dis Child 2013;98:784-6.

24. Mehta DV. Magnetic resonance imaging in paediatric spinal dysraphism with comparative usefulness of various magnetic resonance sequences. J Clin Diagn Res 2017;11:TC17-22.

25. Lee JY, Park SH, Chong S, Phi JH, Kim SK, Cho BK, et al. Congenital dermal sinus and limited dorsal myeloschisis: "spectrum disorders" of incomplete dysjuction between cutaneous and neural ectoderms.
Neurosurgery 2018 Mar 29 [Epub].

26. Albert GW. Spine ultrasounds should not be routinely performed for patients with simple sacral dimples. Acta Paediatr 2016;105:890-4.

27. Zywicke HA, Rozzelle CJ. Sacral dimples. Pediatr Rev 2011;32:10913.

28. Scottoni F, Iacobelli BD, Zaccara AM, Totonelli G, Schingo AM, Bagolan P. Spinal ultrasound in patients with anorectal malformations: is this the end of an era? Pediatr Surg Int 2014;30:829-31. 